\title{
Helminth infection-induced carcinogenesis: spectrometric insights from the liver flukes, Opisthorchis and Fasciola
}

\author{
Maria João Gouveia ${ }^{1,2,3}$, Maria Y. Pakharukova ${ }^{4,5}$, Gabriel Rinaldi ${ }^{6}$, Viatcheslav A. Mordvinov ${ }^{4}$, \\ Paul J. Brindley ${ }^{6}$, Fátima Gärtner ${ }^{1,3,7}$ and Nuno Vale $3,7,8, \star$ (D) \\ ${ }^{1}$ Department of Molecular Pathology and Immunology, Institute of Biomedical Sciences Abel Salazar (ICBAS), University of \\ Porto, Rua de Jorge Viterbo Ferreira 228, 4050-313 Porto, Portugal, ${ }^{2}$ Center for the Study of Animal Science, CECA-ICETA, \\ University of Porto, Praça Gomes Teixeira, Apartado 55142, 4051-401 Porto, Portugal, ${ }^{3}$ i3S, Instituto de Investigação e Inovação \\ em Saúde, University of Porto, Rua Alfredo Allen, 208, 4200-135 Porto, Portugal, ${ }^{4}$ Laboratory of Molecular Mechanisms of \\ Pathological Processes, Institute of Cytology and Genetics, Siberian Branch of the Russian Academy of Science, 10 Lavrentiev \\ Avenue, 630090 Novosibirsk, Russia, ${ }^{5}$ Department of Natural Sciences, Novosibirsk State University, 2 Pirogov Street, 630090 \\ Novosibirsk, Russia, ${ }^{6}$ Department of Microbiology, Immunology \& Tropical Medicine, and Research Center for Neglected \\ Diseases of Poverty, School of Medicine \& Health Sciences, George Washington University, Washington, D.C., 20037, USA, \\ ${ }^{7}$ Institute of Molecular Pathology and Immunology of the University of Porto (IPATIMUP), Rua Júlio Amaral de Carvalho \\ 45, 4200-135 Porto, Portugal, and ${ }^{8}$ Laboratory of Pharmacology, Department of Drug Sciences, Faculty of Pharmacy, University \\ of Porto, Rua de Jorge Viterbo Ferreira 228, 4050-313 Porto, Portugal \\ Current address: Gabriel Rinaldi, Wellcome Sanger Institute, Wellcome Genome Campus, Hinxton, Cambridge CB10 1SA, UK \\ ${ }^{\star}$ Corresponding author: Email: nuno.vale@ff.up.pt (N. Vale)
}

(Received 26 May 2020; Revised 22 July 2020; Accepted 23 July 2020)

\begin{abstract}
Earlier reports revealed oxysterol metabolites of Opisthorchis spp. liver fluke origin conjugated with DNA bases, suggesting that the generation of these DNA-adducts may underlie the mutagenicity and carcinogenicity of the infection with these food-borne pathogens. Here, we employed liquid chromatography-mass spectrometry to investigate, compare and contrast spectrograms of soluble extracts from Fasciola hepatica adult worms from bile ducts of cattle with those from O. viverrini and O. felineus from experimentally infected hamsters. F. hepatica and Opisthorchis spp. shared common compounds including oxysterol-like metabolites, bile acids and DNA-adducts, but the spectrometric profiles of $F$. hepatica included far fewer compounds than Opisthorchis species. These findings support the postulate that parasitic oxysterol-like metabolites could be related to carcinogenesis associated to infection and they point to a molecular basis for the differences among major groups of liver flukes concerning infection-induced malignancy.
\end{abstract}

Keywords: Fasciola hepatica; Opisthorchis viverrini; Opisthorchis felineus; oxysterols; DNA adducts

\section{Introduction}

More than $20 \%$ of cancer in the developing world are caused by infections (Brindley et al., 2015). The World Health Organization's International Agency for Research on Cancer (IARC) recognizes the infection with about 12 pathogens as group 1 biological carcinogens, i.e., definitive causes of cancer. These group 1 agents include three helminth parasites, specifically the fish-borne trematodes (FBT) Opisthorchis viverrini and Clonorchis sinensis and the blood fluke, Schistosoma haematobium (IARC, 2012). In addition, we have previously reported findings from hamster infection that support the inclusion of Opisthorchis felineus, also an FBT, to this list of biological carcinogens and definitive cause

\footnotetext{
(c) The Author(s), 2020. Published by Cambridge University Press. This is an Open Access article, distributed under the terms of the Creative Commons Attribution licence (http://creativecommons.org/licenses/by/4.0/), which permits unrestricted re-use, distribution, and reproduction in any medium, provided the original work is properly cited.
} 
of cholangiocarcinoma (Gouveia et al., 2017). We hypothesized that these helminths produce and release derivatives of oestrogens and oxysterols that promote oxidation of host DNA and have the ability of parasite metabolites to directly promote DNA lesions adducts and mutations that ultimately lead to cholangiocarcinoma (Brindley et al., 2015; Costa et al., 2014; Gouveia et al., 2015; 2017; Vale et al., 2013). The findings supported the postulate that these infection-associated cancers originate from a biological and/ or chemical insult followed by chronic inflammation, fibrosis, and a change in the tissue microenvironment that leads to a pre-cancerous niche (Brücher \& Jamall, 2014; Cavalieri et al., 2017). Paradoxically, infections with other close phylogenetic relatives of these carcinogenic helminths, also food borne trematodes of the Phylum Platyhelminthes, have not been categorized as group 1 biological carcinogens (Chapman et al., 1999; Montero et al., 1999; Kawanishi et al., 2006; Kolodziejczyk et al., 2006; Machicado et al., 2016; Mayer \& Fried, 2007; Tsocheva-Gaytandzhieva, 2005).

For instance, Fasciola hepatica has a wide geographical range, causes major economic loss in sheep and cattle worldwide, and also is an important zoonosis in humans (Villegas et al., 2012). It has been shown that fascioliasis can induce host DNA damage through action of reactive nitric species (RNS) or oxygen species (ROS) (Mayer \& Fried, 2007; Tsocheva et al., 1992); however, the infection has not been associated to carcinogenesis. Seeking new insights in this apparent paradox among closely related foodborne trematodes, here we conducted an analysis of soluble extracts of adult worms of $F$. hepatica, O. viverrini and $O$. felineus by liquid chromatography coupled with mass spectrometry (LC-MS/MS). Remarkably, the LC-MS/MS chromatograms for each of the liver fluke species exhibited clear differences in regard the presence of oxysterols. These metabolites were minor components of the extract from F. hepatica, in contrast to the relatively high abundance and diversity of oxysterols in O. viverrini and O. felineus. The presence of abundant oxysterols in the of Opisthorchis liver flukes would support the hypothesis that these molecules may act as initiators during the liver fluke infection-induced biliary tract malignancy.

\section{Material}

\section{Ethics Statement}

Procedures undertaken complied with The Code of Ethics of the World Medical Association (Declaration of Helsinki) for animal experiments http://ec.europa.eu/environment/chemicals/lab_animals/legislation_ en.htm. Syrian hamsters (Mesocricetus auratus) were purchased from the stock of the Puschino Animal Facility (Russia) and bred at the Animal Facility of the ICG SB RAS (RFMEFI61914X0005) (Russia). The hamsters were maintained according to protocols approved by the Committee on the Ethics of Animal Experiments of the Institute of Cytology and Genetics (Permit Number: 25 of 12.12.2014).

\section{Soluble extracts from $\mathrm{F}$. hepatica, $\mathrm{O}$. viverrini and $\mathrm{O}$. felineus adult liver flukes}

Adult worms of $F$. hepatica were obtained from the bile ducts of infected cattle at a local slaughterhouse (Silva et al., 2004). It should be noted that the animals were processed as part of normal work of the slaughterhouse. O. viverrini and O. felineus were obtained as previously described (Gouveia et al., 2017; Vale et al., 2013). In brief, metacercariae of Opisthorchis species were obtained from naturally infected cyprinoid fish in Khon Kaen province, Thailand or from naturally infected fish (Leuciscus idus) in the Ob River near the city of Novosibirsk, Siberia Russia, respectively. The fish were digested with pepsin-HCl (Gouveia et al., 2017). Fifty metacercariae were used to infect hamsters (Mesocricetus auratus) and three months after infection, the animals were euthanized and adult $O$. viverrini or $O$. felineus flukes recovered from their bile ducts. The worms were washed extensively in phosphate buffered saline (PBS, $\mathrm{pH}$ 7.4) supplemented with $100 \mu \mathrm{g} / \mathrm{ml}$ streptomycin and $100 \mathrm{U} / \mathrm{ml}$ penicillin $\mathrm{G}$ and cultured overnight in serum free RPMI-1640 medium (Lonza, Basel, Switzerland) containing 1\% glucose, and protease inhibitors (0.1 $\mathrm{mM}$ phenylmethanesulfonyl fluoride, $2 \mu \mathrm{M}$ E-64 and $10 \mu \mathrm{M}$ leupeptin) (Sigma-Aldrich, St. Louis, Missouri) at $37^{\circ} \mathrm{C}, 5 \% \mathrm{CO}_{2}$. 
Soluble extracts from all samples were prepared by sonication ( 5 x $5 \mathrm{~s}$ burst, output cycle 4 , Branson Sonifier 450, Germany) in PBS supplemented with protease inhibitors [500 $\mu \mathrm{M} 4$-(2-aminoethyl) benzenesulfonyl fluoride hydrochloride (AEBSF), $0.3 \mu \mathrm{M}$ aprotinin, $10 \mu \mathrm{M}$ E-64, $10 \mu \mathrm{M}$ bestatin and $10 \mu \mathrm{M}$ leupeptin] (M221, Amresco, Solon, OH, USA), followed by $30 \mathrm{~min}$ centrifugation at $10,000 \mathrm{rpm}, 4^{\circ} \mathrm{C}$. The protein concentration of supernatants was determined using a commercial kit. Ascorbic acid was added to $1 \mathrm{mg} / \mathrm{ml}$ to these extracts, which were stored in aliquots at $-80^{\circ} \mathrm{C}$ (Gouveia et al., 2017; Vale et al., 2013).

\section{Sample preparation and LC-MS/MS analysis}

Samples were prepared and processed using liquid chromatography diode array detection electron spray ionization mass spectrometry, as previously described (Gouveia et al., 2015; 2017; Vale et al., 2013). Due to the acceptable chromatographic performance of methanol as the solvent in terms of separation and sensitivity, with short gradient times (Wang et al., 2000), this solvent was added up to $20 \%$ (v/v). High performance liquid chromatography coupled with mass spectrometer was employed to investigate molecular species from liver flukes, with samples of $25 \mu \mathrm{l}$ injected into the LC-MS/MS instrument for analysis. The mass analysis was performed within an LTQ Orbitrap XL mass spectrometer (Thermo Fischer Scientific, Bremen, Germany), fitted with an ultraviolet (UV) photo diode array (PDA) detector. Analysis involved a Macherey-Nagel Nucleosil C18-column $(250 \mathrm{~mm}$ x $4 \mathrm{~mm}$ internal diameter; $5 \mu \mathrm{m}$ particle diameter, end-capped), proceeding at a flow rate of $0.3 \mathrm{ml} / \mathrm{min}$. The capillary voltage of the electrospray ionization was $28 \mathrm{~kW}$, capillary temperature was $310^{\circ} \mathrm{C}$, flow rates of the sheath gas and auxiliary $\mathrm{N} 2$ were set to 40 and 10 (arbitrary unit as provided by the software settings), respectively, and gas temperature was $275^{\circ} \mathrm{C}$ (Gouveia et al., 2015; 2017; Vale et al., 2013). The mobile phase consisted of $1 \%$ formic acid in water (A)/acetonitrile (B) mixtures. Eluates were monitored for $75 \mathrm{~min}$, run with a mobile phase gradient of $0-5 \mathrm{~min}, 100 \% \mathrm{~A} ; 5-10 \mathrm{~min}$, linear gradient from $100 \%$ to $80 \% \mathrm{~A}, 10-15 \mathrm{~min}$ $80 \%$ A, $15-50 \mathrm{~min}$, linear gradient from $80 \%$ to $40 \% \mathrm{~A} ; 50-65 \mathrm{~min}, 40 \% \mathrm{~A} ; 65-75 \mathrm{~min}$, linear gradient from $40 \%$ to $100 \% \mathrm{~B}$. Washing for $15 \mathrm{~min}$ with acetonitrile was carried out to stabilize the column. Data were collected in negative electrospray ionization negative mode scanning a mass to charge ratio $(\mathrm{m} / \mathrm{z})$ range of $50-2,000$.

\section{Results}

\section{Both species of Opisthorchis shared identical mass spectra profiles}

We have developed a sensitive LC-MS/MS-based protocol to identify new steroids-derived molecules not only in extracts of helminth parasites (Gouveia et al., 2017; Vale et al., 2013), but also from experimental infected rodents (Gouveia et al., 2017) and naturally-infected humans (Gouveia et al., 2015). Extracts obtained from $F$. hepatica adult worms were analyzed in order to provide insights related to their composition and complexity.

Comparing data obtained for O. viverrini with O. felineus we observed that both these liver flukes displayed highly similar mass spectra (MS) and shared most peaks detected (indicated in grey in Fig 1) which were attributed to oxysterol-like metabolites, e.g. mass/charge $(\mathrm{m} / \mathrm{z}) 356,307$, bile acids in oxidized form, e.g. m/z 443, 479, 488 and DNA-adducts, e.g. m/z 599, 639, 667 (Gouveia et al., 2017; Vale et al., 2013).

\section{F. hepatica extracts exhibited striking differences to those of the Opisthorchis species}

Notable differences were apparent among the MS profiles of $F$. hepatica and the Opisthorchis species. Most of compounds present in both Opisthorchis species were absent from F. hepatica, specifically $\mathrm{m} / \mathrm{z}$ $356,357,425$ and 307 . Remarkably, these specific compounds were attributed to be oxysterols with ability to react with host DNA as described (Gouveia et al., 2017). The MS profile of $F$. hepatica was much more 
a) Common $\mathbf{m} / \mathbf{z}$

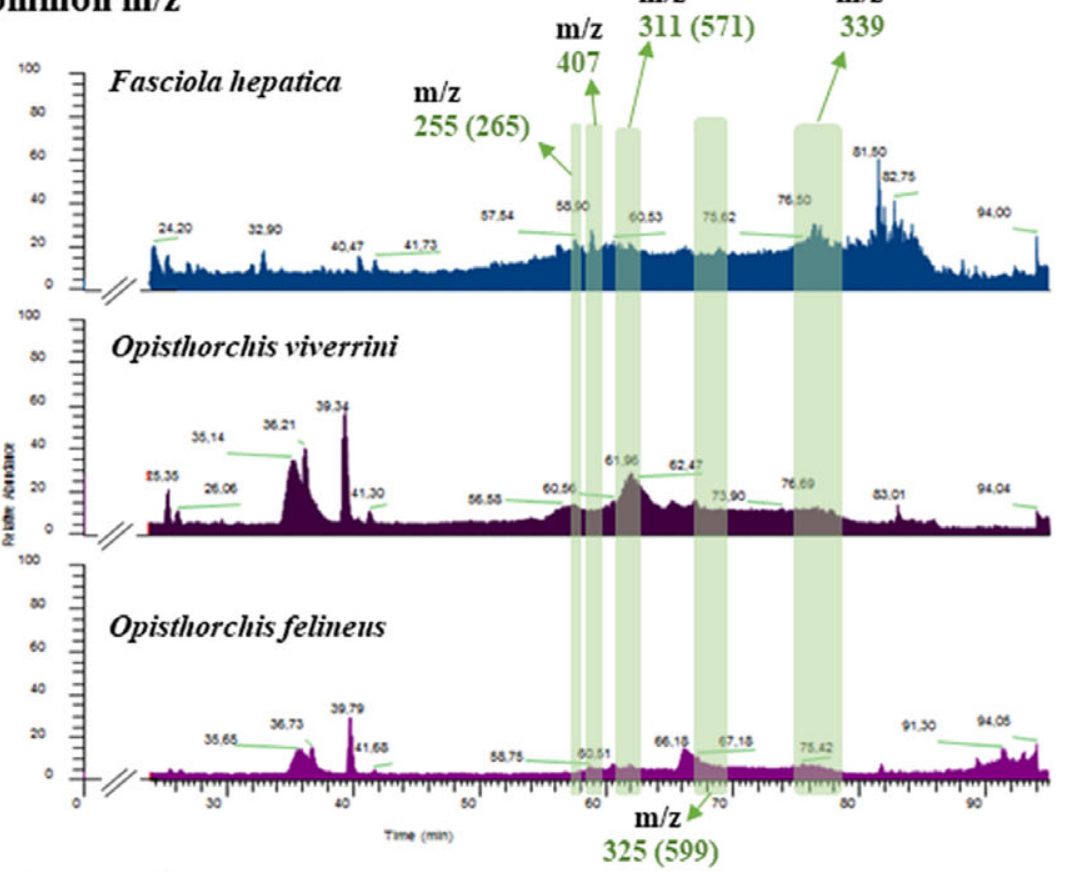

b) Different $\mathbf{m} / \mathbf{z}$

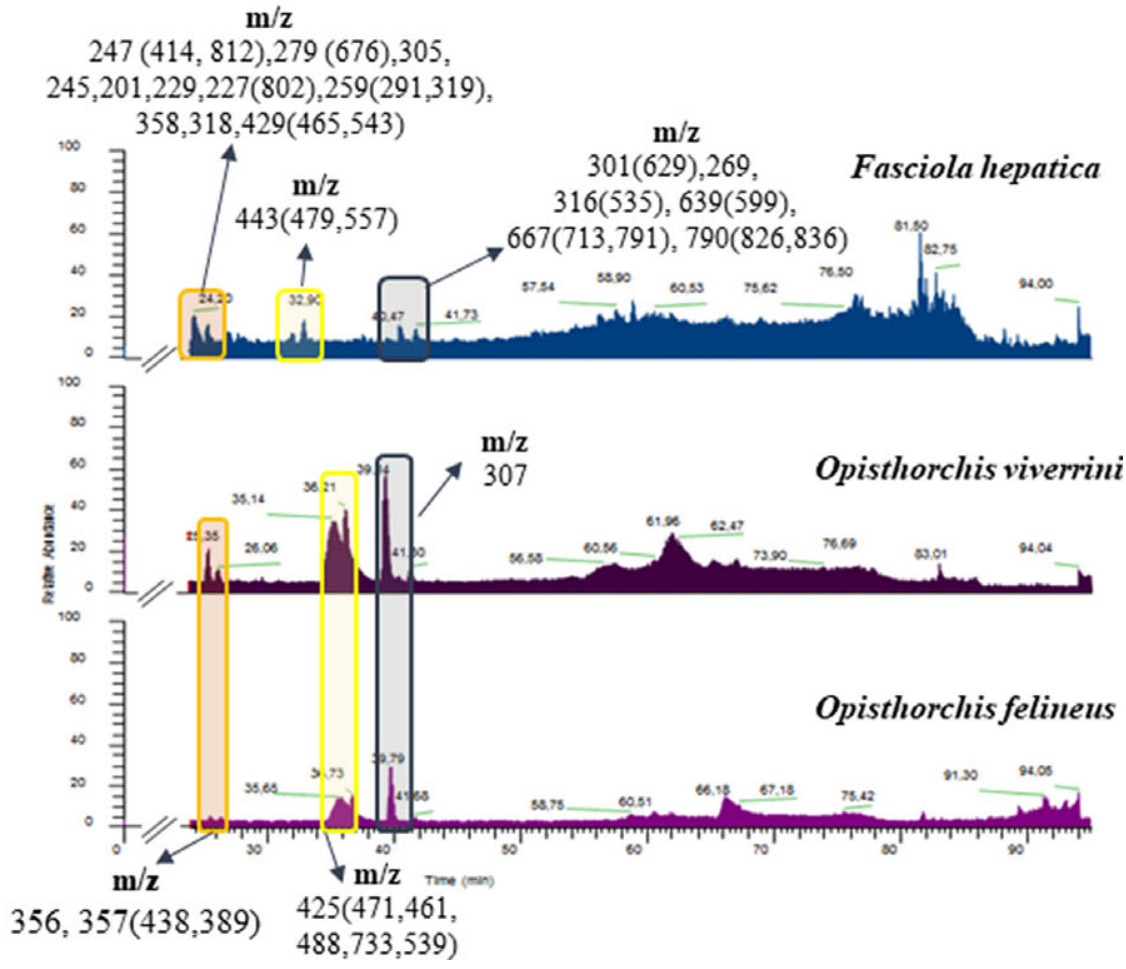

Fig. 1 Comparison of mass spectral profiles obtained for Fasciola hepatica and Opisthorchis spp. Panel A, common m/z between the three liver flukes; panel $\mathrm{B}$, major differences among the liver flukes. 
complex than those obtained for Opisthorchis spp. (Fig. 1). The major differences were observed at retention intervals of approximately 24,32 , and $40 \mathrm{~min} \mathrm{-} \mathrm{as} \mathrm{indicated} \mathrm{in} \mathrm{orange,} \mathrm{yellow} \mathrm{and} \mathrm{blue,}$ respectively, on the chromatographs (Fig. 1). On these retention times, F. hepatica showed greater number of compounds in comparison to those observed on Opisthorchis species (Fig. 1 and Table 1). Remarkably, most of these compounds were detected only in F. hepatica extracts (Table 1).

Unlike Opisthorchis, F. hepatica displayed a higher number of compounds with elevated $\mathrm{m} / \mathrm{z}$ (between 600 and 800), mostly between retention interval of 38 to $42 \mathrm{~min}$ (Table 1), which might suggest that they are more complex than the majority of those detected on Opisthorchis spp.

Table 1. Comparison of mass/charge $(\mathrm{m} / \mathrm{z})$ obtained for Fasciola hepatica during this study with Opisthorchis spp. previously reported for O. viverrini (Gouveia et al., 2017; Vale et al., 2013) and O. felineus (Gouveia et al., 2017). The structures of common $\mathrm{m} / \mathrm{z}$ (signed at green) are depicted on Table 2.

\begin{tabular}{|c|c|c|c|c|}
\hline Retention time (min) & $\mathrm{m} / \mathrm{z}$ & $F h$ & Ov & of \\
\hline 17.64 & 337.08 & $\checkmark$ & $\checkmark$ & \\
\hline 23.24 & 320.17 & $\checkmark$ & & \\
\hline 23.25 & 353.13 & $\checkmark$ & & \\
\hline 23.95 & 293.12 & $\checkmark$ & & \\
\hline \multirow[t]{2}{*}{24.17} & 245.12 & $\checkmark$ & & \\
\hline & 414.22 & $\checkmark$ & & \\
\hline \multirow[t]{2}{*}{24.26} & 279.14 & $\checkmark$ & & \\
\hline & 676.30 & $\checkmark$ & & \\
\hline 24.36 & 305.08 & $\checkmark$ & & \\
\hline 24.41 & 245.12 & $\checkmark$ & & \\
\hline \multirow[t]{2}{*}{24.75} & 229.16 & $\checkmark$ & & \\
\hline & 201.13 & $\checkmark$ & & \\
\hline 25.08 & 227.14 & $\checkmark$ & & \\
\hline \multirow[t]{3}{*}{25.13} & 259.13 & $\checkmark$ & & \\
\hline & 291.10 & $\checkmark$ & & \\
\hline & 319.10 & $\checkmark$ & & \\
\hline 25.41 & 358.20 & $\checkmark$ & & \\
\hline 25.54 & 318.11 & $\checkmark$ & & \\
\hline \multirow[t]{3}{*}{28.12} & 429.23 & $\checkmark$ & & \\
\hline & 465.20 & $\checkmark$ & & \\
\hline & 543.22 & $\checkmark$ & & \\
\hline 32.89 & 443.24 & 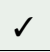 & & \\
\hline 37.65 & 301.07 & $\checkmark$ & & \\
\hline 38.18 & 629.30 & $\checkmark$ & & \\
\hline \multirow[t]{2}{*}{39.50} & 316.17 & $s$ & & \\
\hline & 535.23 & $\checkmark$ & & \\
\hline \multirow[t]{3}{*}{40.50} & 677.50 & 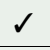 & & \\
\hline & 713.48 & $\checkmark$ & & \\
\hline & 724.51 & $\checkmark$ & & \\
\hline
\end{tabular}


Table 1. Continued

\begin{tabular}{|c|c|c|c|c|}
\hline Retention time (min) & $\mathrm{m} / \mathrm{z}$ & $F h$ & Ov & of \\
\hline \multirow[t]{3}{*}{41.70} & 790.58 & $\checkmark$ & & \\
\hline & 826.56 & $\checkmark$ & & \\
\hline & 837.59 & $\checkmark$ & & \\
\hline 51.02 & 447.14 & $\checkmark$ & & \\
\hline 54.86 & 321.18 & $\checkmark$ & & \\
\hline 58.70 & 255.23 & $\checkmark$ & $\checkmark$ & $\checkmark$ \\
\hline 58.92 & 407.28 & $\checkmark$ & $\checkmark$ & $\checkmark$ \\
\hline 59.93 & 571.29 & $\checkmark$ & $\checkmark$ & $\checkmark$ \\
\hline 61.06 & 311.17 & $\checkmark$ & $\checkmark$ & $\checkmark$ \\
\hline 64.25 & 325.19 & $\checkmark$ & $\checkmark$ & $\checkmark$ \\
\hline 64.15 & 599.32 & $\checkmark$ & $\checkmark$ & $\checkmark$ \\
\hline 70.16 & 367.25 & $\checkmark$ & & \\
\hline 77.95 & 339.20 & $\checkmark$ & $\checkmark$ & $\checkmark$ \\
\hline 76.38 & 391.29 & $\checkmark$ & & \\
\hline 81.63 & 465.31 & $\checkmark$ & & \\
\hline
\end{tabular}

Table 2. Structures of $\mathrm{m} / \mathrm{z}$ common to Fasciola hepatica and Opisthorchis species.

\begin{tabular}{|c|c|c|c|c|c|}
\hline Retention time (min) & $\mathrm{m} / \mathrm{z}$ & $F h$ & Ov & of & Structures \\
\hline 57.54 & 255.07 & $\checkmark$ & $\checkmark$ & $\checkmark$ & \\
\hline 58.92 & 407.28 & $\checkmark$ & $\checkmark$ & $\checkmark$ & \\
\hline 59.93 & 571.29 & $\checkmark$ & $\checkmark$ & $\checkmark$ & \\
\hline
\end{tabular}


Table 2. Continued

\begin{tabular}{|c|c|c|c|c|c|}
\hline Retention time (min) & $\mathrm{m} / \mathrm{z}$ & $F h$ & Ov & of & Structures \\
\hline 61.06 & 311.17 & $\checkmark$ & $\checkmark$ & $\checkmark$ & \\
\hline 64.25 & 325.19 & $\checkmark$ & $\checkmark$ & $\checkmark$ & \\
\hline 64.15 & 599.32 & $\checkmark$ & $\checkmark$ & $\checkmark$ & \\
\hline 77.95 & 339.20 & $\checkmark$ & $\checkmark$ & $\checkmark$ & \\
\hline
\end{tabular}

Nonetheless, F. hepatica and Opisthorchis spp. shared several common compounds at retention interval of 58-64 min (signed by green in Fig. 1 and Table 1). These compounds have been ascribed previously to oxysterol-like metabolite (e.g. m/z 325), bile acids (e.g. m/z 571) and as well as DNAadducts (m/z 599) (Vale et al., 2013) which is expected since parasites reside on bile ducts. Remarkably, however, oxysterol-like metabolites, bile acids and DNA-adducts in particular were fewer in F. hepatica compared to Opisthorchis spp. The fact, F. hepatica has lesser oxysterol-like metabolites might be one of the reasons that explain why a carcinogenic potential associated with its infection has not been established.

\section{Discussion}

Both chronic infection with Fasciola spp. and Opisthorchis spp. could lead to fibrosis, hyperplasia, and biliary stasis (Gouveia et al., 2017; Machicado et al., 2016; Maksimova et al., 2017; Motorna et al., 2001; Sithithaworn et al., 2012). However, an association between fascioliasis and cancer remains controversial and not definitely established (Machicado et al., 2016). Thus, we decided to investigate extracts of adult 


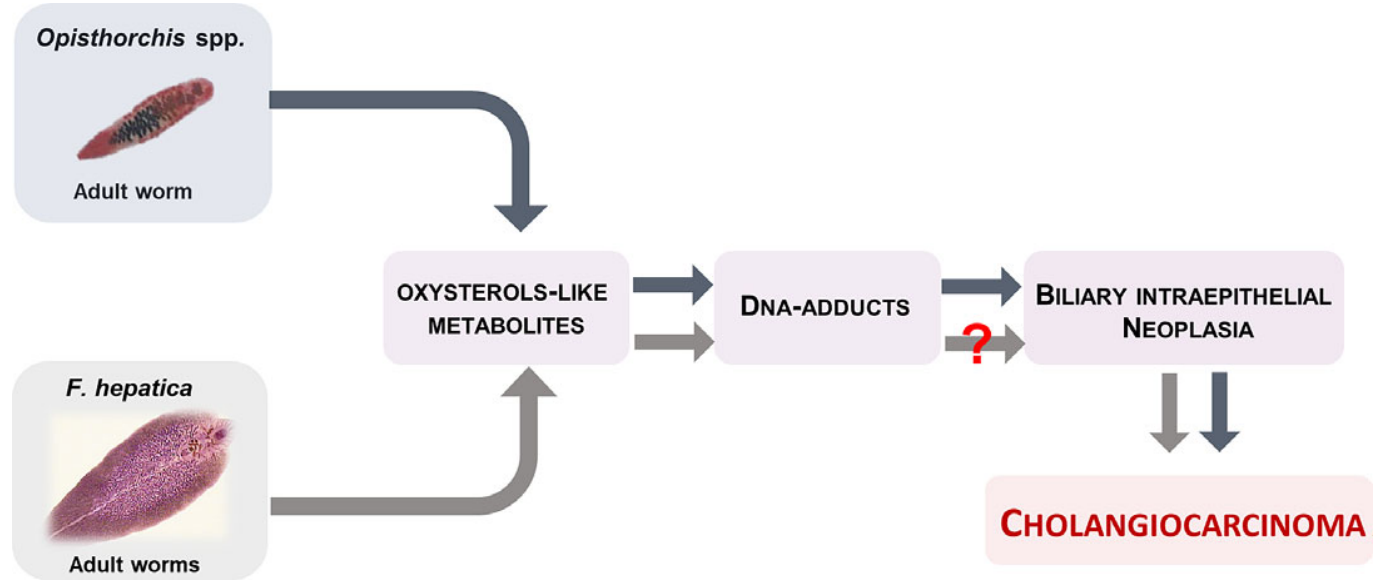

Fig. 2 Adult liver flukes 0 . viverrini and 0 . felineus produces oxysterol-like metabolites that interact with host chromosomal DNA to form DNA-adducts and forms of biliary intraepithelial neoplasia that conducive to cholangiocarcinoma. F. hepatica also elaborates oxysterol-like metabolites, but at much lower number, which might be explain, at least in part, why infection with this parasite fails to induce malignancy.

worms of F. hepatica and compare with data previously obtained for Opisthorchis spp. We aimed to address the following questions: 1 ) does $F$. hepatica synthesize and excrete metabolites that might promote direct damage on host DNA? The MS profile of $F$. hepatica was found to be far more complex, showing an elevated number of compounds more complex that translate in an elevated $\mathrm{m} / \mathrm{z}$ rather than Opisthorchis spp. This suggested that metabolic process that occur in F. hepatica are dissimilar to those in Opisthorchis spp. and also could be related to its complex route of migration to the biliary tract might underlie these differences.

Compounds of $F$. hepatica might be related to the different migratory route of the parasite to the biliary tree. Unlike Opisthorchis spp., newly excysted juveniles of F. hepatica exit the lumen of the small intestine, transverse the intestinal wall and migrate through the abdominal cavity to the Glisson's capsule of the liver (Mas-Coma, 2005; Moazeni \& Ahmadi, 2016). This parasite might deploy more complex biochemical processes and secretions, including the secretion of cathepsins (Cancela et al., 2010; Cwiklinski et al., 2015; Young et al., 2010) to accomplish this elaborate organ and tissue migration. The juvenile F. hepatica infects the liver by directly penetrating the Glisson's capsule from the abdominal cavity, and thereafter burrows through the hepatic parenchyma to the bile ducts where it eventually matures into the egg-laying adult worm (Moazeni \& Ahmadi, 2016). Components detected in the extracts of $F$. hepatica might be related with digestion of host tissues including blood such as hemoglobin, albumin and immunoglobin to support reproductive process including synthesis of eggs (Moazeni \& Ahmadi, 2016). On other hand, most of the compounds observed from 23 to 57 minutes were specific of F. hepatica, i.e. not present in Opisthorchis. Juvenile Opisthorchis flukes ascend from the duodenum directly into the lumen of biliary tree (Sithithaworn et al., 2012; Pakharukova et al., 2016).

Glycocholic acid in the mammalian small intestine triggers the excystment of the metacercaria and emergence of $F$. hepatica juvenile flukes stimulating the exit of the parasite from the gut lumen and its migration to the abdominal cavity. Intriguingly, the juvenile $F$. hepatica did not survive in bile-containing solutions whereas the adult fluke resides in the bile ducts, bathed in bile (Tielens et al., 1981). Differences in the nature of the juvenile versus adult tegument of $F$. hepatica and the selectivity and the permeability of glycocalyx of the tegument may underpin these stage specific differences (Tielens et al., 1981). The complexity of the tegument, a complex metabolically active and highly glycosylated biological matrix (Ravidá et al., 2016) might also underpin complexity of F. hepatica MS profile and its components.

Some of these compounds might be precursors of known compounds that were previously attributed to oxysterol-like metabolites, bile acids and DNA-adducts (Gouveia et al., 2017; Vale et al., 2013). This is 
feasible since all three flukes live within the biliary tree. There is evidence that $F$. hepatica induces DNA damage through the action of mutational mediators (Jedina et al., 2011; Kolodziejczyk et al., 2006). The presence of DNA adducts in tissue does not necessarily imply a specific tumorigenic risk for the host tissue. Other factors such as DNA repair and cell proliferation key roles players in determining the overall carcinogenic risk (Povey, 2000). An association between fascioliasis and cancer has only been suggested from in vitro studies and, thus far, there have not been satisfactory reports of human cases of bile duct cancer due to chronic infection with F. hepatica (Chun et al., 2012; Gentile et al., 1998; Hanahan \& Weinberg, 2011; Machicado et al., 2016; Tsocheva-Gaytandzhieva, 2005). Therefore, there is a lack of cogent evidence that relate fascioliasis with cancer (Machicado et al., 2016). By contrast, a number of reports posit opposing effects, i.e. tumor growth stimulation and inhibition. Tumor growth stimulation and proliferation of hepatocytes has been observed during acute phase of infection where larval flukes migrate through the parenchyma of the liver and provoke marked inflammation (Montero et al., 1999; Tsocheva et al., 1992). In turn, the chronic inflammation increases oxidative stress that can overwhelm antioxidant system homeostasis to dampen reactive oxygen species and consequent oxidative modification of lipids, nucleic acids and proteins (Kolodziejczyk et al., 2006). Like fascioliasis, opisthorchiasis is characterized by elevated oxidative stress and altered the antioxidant systems (Kawanishi et al., 2006; Kolodziejczyk et al., 2006). We also documented that infection with O. felineus induces biliary intraepithelial neoplasia (BilIN). The consonance of findings that the presence of new metabolites and of BilIN-1 and BilIN-2 indicates that $O$. felineus infection induces neoplastic transformation of cholangiocytes and can be expected to promote growth of biliary cancers (Gouveia et al., 2017). Tumor inhibition has been noted during the chronic phase of fascioliasis that may dampen the liver metabolizing activity (Montero et al., 1999). Whereas acute $F$. hepatica infection may increase the metabolizing enzymes in liver and thus increase the activation of exogenous carcinogens (Motorno et al., 2001), chronic infection may reduce hepatic metabolizing activity (Montero et al., 1999). It is noteworthy that chronic infection with $F$. hepatica in a rat model suppressed $N$-nitrosodimethyldiamine-induced carcinogenesis, suggesting a parasite-induced inhibition of carcinogenesis in the liver of rodents experimentally infected with F. hepatica (Tsocheva et al., 1992). Could this ability be one of the reasons why the infection with F. hepatica does not present carcinogenic potential? Is F. hepatica able to neutralize the reactivity of oxysterol-like molecules or other carcinogenic with host DNA? All these questions require further investigation. This study aimed to characterize the differences between Fasciola and Opisthorchis with a view to identifying the parasite-derived compound that results in the different pathogenic outcomes after infection with these two parasites. Not only is this important to understand mechanisms underlying the pathology, with a view to perhaps developing appropriate therapeutics in the future, but it would also provide information to understand how two flukes have adapted to induce such different outcomes in their hosts. Future studies will evaluate if there is a host-related effect on the production of the metabolites identified in this study.

In conclusion, we discovered that Fasciola hepatica displayed more complex mass spectra profile that the Opisthorchis species and several specific compounds that might be related to its complex route of migration to the biliary tract. Nonetheless, F. hepatica shared few compounds with Opisthorchis species, which are related to oxysterols, bile acids and DNA-adducts. The presence of only a few common compounds might explain why fascioliasis has not been causally linked with liver cancer. Indeed, we posit that presence of fewer oxysterol-like metabolites might (partially) explain why definitive carcinogenic potential has not been ascribed to ruminant or human fascioliasis (Fig. 2). More study can be expected to enhance our understanding of the differences in carcinogenicity between these two genera of food borne liver flukes.

Acknowledgments. NV thanks Fundação para a Ciência e a tecnologia (FCT, Portugal) and Fundação Manuel António da Mota (FMAM, Portugal) by support Nuno Vale Lab. The contents of this report are solely the responsibility of the authors and do not necessarily represent the official views of the FCT or FMAM.

Author Contributions. M.J.G. and N.V. conceived and planned the experiments. M.J.G., M.P. and N.V. carried out the experiments. M.J.G. contributed to sample preparation. M.J.G., M.P., G.R., P.B. and N.V. contributed to the interpretation of 
the results. M.J.G. and N.V. took the lead in writing the manuscript. All authors provided critical feedback and helped shape the research, analysis and manuscript.

Funding Information. This work was financed by FEDER - Fundo Europeu de Desenvolvimento Regional funds through the COMPETE 2020 - Operacional Programme for Competitiveness and Internationalisation (POCI), Portugal 2020, and by Portuguese funds through FCT - Fundação para a Ciência e a Tecnologia, in the framework of the project, Institute for Research and Innovation in Health Sciences" (POCI-01-0145-FEDER-007274). The FCT and FEDER (European Union) also supported these studies through project number IF/00092/2014/CP1255/CT0004. This work was also supported in part by the Russian Science Foundation [grant number 18-15-00098 (VAM)].

Conflict of Interest. On behalf of all authors, the corresponding author states that there is no conflict of interest.

Data availability. The authors confirm that the data supporting the findings of this study are available within the article.

\section{References}

Brindley, P. J., Costa, J. M. C., \& Sripa, B. (2015). Why does infection with some helminths cause cancer?. Trends in Cancer, 1, 174-182.

Brücher, B. L., \& Jamall, I. S. (2014). Epistemology of the origin of cancer: A new paradigm. BMC Cancer, 14, 331.

Cancela, M., Ruétalo, N., Dell'Oca, N., da Silva, E., Smircich, P., Rinaldi, G., Roche, L., Carmona, C., Alvarez-Valín, F., Zaha, A., \& Tort, F. (2010). Survey of transcripts expressed by the invasive juvenile stage of the liver fluke Fasciola hepatica. BMC Genomics, 11, 227.

Cavalieri, E. L., Rogan, E. G., \& Zahid, M. (2017). Critical depurinating DNA adducts: Estrogen adducts in the etiology and prevention of cancer and dopamine adducts in the etiology and prevention of Parkinson's disease. International Journal of Cancer, 141, 1078-1090.

Chapman, R. W. (1999). Risk factors for biliary tract carcinogenesis. Annual Oncology, 10, 308-311.

Chun, J. Y., Bae, Y. A., Yun, D. H., Yang, H. J., \& Kong, Y. (2012). Experimental murine fascioliasis derives early immune suppression with increased levels of TGF- $\beta$ and IL-4. Korean Journal of Parasitology, 50, 301-308.

Costa, J. M. C., Vale, N., Gouveia, M. J., Botelho, M. C., Sripa, B., Santos, L. L., Santos, J. H., Rinaldi, G., \& Brindley, P. J. (2014). Schistosome and liver fluke catechol-estrogens and helminth associated cancers. Frontiers in Genetics, 5, 444.

Cwiklinski, K., Dalton, J. P., Dufresne, P. J., La Course, J., Williams, D. J. L., Hodgkinson, J., \& Paterson, S. (2015). The Fasciola hepatica genome: Gene duplication and polymorphism reveal adaptation to the host environment and the capacity for rapid evolution. Genome Biology, 16, 71.

Gentile, J. M., Gentile, G. J., Nannenga, B., Johnson, M., Blankespoor, H., \& Montero, R. (1998). Enhanced liver cell mutations in trematode-infected Big Blue transgenic mice. Mutation Research/Fundamental Molecular Mechanism of Mutagenesis, 400, 355-360.

Gouveia, M. J., Pakharukova, M. Y., Laha, T., Sripa, B., Maksimova, G. A., Rinaldi, G., Mordvinov, V. A., Amaro, T., Santos, L. L., Costa, J. M. C., \& Vale, N. (2017). Infection with Opisthorchis felineus induces intraepithelial neoplasia of the biliary tract in a rodent model. Carcinogenesis, 38, 929-937.

Gouveia, M. J., Santos, J., Brindley, P. J., Rinaldi, G., Lopes, C., Santos, L. L., da CostaJ. M., \& Vale, N. (2015). Estrogen-like metabolites and DNA-adducts in urogenital schistosomiasis-associated bladder cancer. Cancer Letters. 359, $226-232$.

Hanahan, D., \& Weinberg, R. A. (2011). Hallmarks of cancer: The next generation. Cell, 144, 646-674.

International Agency for Research on Cancer (IARC). (2012). Biological agents. A review of human carcinogens. IARC monographs on the evaluation of carcinogenic risks to humans/World Health Organization. International Agency Research on Cancer, 100B, 1-441.

Jedina, L., Kozak-Ljunggren, M., \& Wedrychowicz, H. (2011). In vivo studies of the early, peritoneal, cellular and free radical response in rats infected with Fasciola hepatica by flow cytometry analysis. Experimental Parasitology, 128, $291-297$.

Kawanishi, S., Hiraku, Y., Pinlaor, S., \& Ma, N. (2006). Oxidative and nitrative DNA damage in animals and patients with inflammatory disease in relation to inflammation-related carcinogenesis. Biological Chemistry, 387, 365-372.

Kolodziejczyk, L., Siemieniuk, E., \& Skrzydlewska, E. (2006). Fasciola hepatica: Effects on the antioxidative properties and lipid peroxidation of rat serum. Experimental Parasitology, 113, 43-48.

Machicado, C., Machicado, J. D., Maco, V., Terashima, A., \& Marcos, L. A. (2016). Association of Fasciola hepatica infection with liver fibrosis, cirrhosis, and cancer: A systematic review. PLoS Neglected Tropical Diseases, 10, e0004962.

Maksimova, G. A., Pakharukova, M. Y., Kashina, E. V., Zhukova, N. A., Kovner, A. V., Lvova, M. N., Katokhin, A. V., Tolstikova, T. G., Sripa, B., \& Mordvinov, V. A. (2017). Effect of Opisthorchis felineus infection and dimethylnitrosamine administration on the induction of cholangiocarcinoma in Syrian hamsters. Parasitology International, 66, 458-463.

Mas-Coma, S. (2005). Epidemiology of fascioliasis in human endemic areas. Journal of Helminthology, 79, $207-216$.

Mayer, D. A., \& Fried, B. (2007). The role of helminth infections in carcinogenesis. Advances in Parasitology, 65, $239-296$.

Moazeni, M., \& Ahmadi, A. (2016). Controversial aspects of the life cycle of Fasciola hepatica. Experimental Parasitology, 169, 81-89. 
Montero, R., Gentile, G. J., Frederick, L., McMannis, J., Murphy, T., Silva, G., Blankespoor, H., \& Gentile, J. M. (1999). Induced expression of CYP2A5 in inflamed trematode infested mouse liver. Mutagenesis, 14, 217-220.

Motorna, O. O., Martin, H., Gentile, G. J., \& Gentile, J. M. (2001). Analysis of lacI mutations in Big Blue transgenic mice subjected to parasite-induced inflammation. Mutation Research, 484, 69-76.

Pakharukova, M. Y., \& Mordvinov, V. A. (2016). The liver fluke Opisthorchis felineus: Biology, epidemiology and carcinogenic potential. Transactions of the Royal Society of Tropical Medicine Hygiene, 110, 28-36.

Povey, A. C. (2000). DNA-adducts: Endogenous and induced. Toxicologic Pathology, 28, 405-424.

Ravidá, A., Cwiklinski, K., Aldridge, A. M., Clarke, P., Thompson, R., Gerlach, J. Q., Kilcoyne, M., Hokke, C. H., Dalton, J. P., \& O'Neill, S. M. (2016). Fasciola hepatica surface tegument: Glycoproteins at the interface of parasite and host. Molecular \& Cellular Proteomics, 15, 3139-3153.

Silva, E., Castro, A., Lopes, A., Rodrigues, A., Dias, C., Conceição, A., Alonso, J., Correia da Costa, J. M., Bastos, M., Parra, F., Moradas-Ferreira, P., \& Silva, M. (2004). A recombinant antigen recognized by Fasciola hepatica-infected hosts. Journal of Parasitol, 90, 746-751.

Sithithaworn, P., Andrews, R. H., Nguyen, V. D., Wongsaroj, T., Sinuon, M., Odermatt, P., Nawa, Y., Liang, S., Brindley, P. J., \& Sripa, B. (2012). The current status of opisthorchiasis and clonorchiasis in the Mekong Basin. Parasitology International, 61, 10-16.

Tielens, A. G. M., Van der Meer, P., \& Van den Bergh, S. G. (1981). Fasciola hepatica: Simple, large-scale, in vitro excystment of metacercariae and subsequent isolation of juvenile and liver flukes. Experimental Parasitology, 51, 8-12.

Tsocheva-Gaytandzhieva, N. T. (2005). Fasciolosis and tumour growth. Helminthologia, 42, 107-113.

Tsocheva, N. T., Kadiiska, M. B., Poljakova-Krusteva, O. T., Krustev, L. P., Yanev, S. S., \& Stoytchev, T. S. (1992). Combined effect of fascioliasis and diethylnitrosamine carcinogenesis on activity of the rat liver monooxygenase system. Comparative Biochemistry and Physiology C, 101, 475-479.

Vale, N., Gouveia, M. J., Botelho, M. C., Sripa, B., Suttiprapa, S., Rinaldi, G., Gomes, P., Brindley, P. J., \& Correia da Costa, J. M. (2013). Carcinogenic liver fluke Opisthorchis viverrini oxysterols detected by LC-MS/MS survey of soluble fraction parasite extract. Parasitology International, 62, 535-542.

Villegas, F., Angles, R., Barrientos, R., Barrios, G., Valero, M. A., Hamed, K., Grueninger, H., Ault, S. K., Montresor, A., Engels, D., Mas-Coma, S., \& Gabrielli, A. F. (2012). Administration of triclabendazole is safe and effective in controlling fascioliasis in an endemic community of the Bolivian Altiplano. PLoS Neglected Tropical Diseases, 6, e1720.

Wang, K. X., Shibue, T., \& Gross, M. L. (2000). Non-covalent complexes between DNA-binding drugs and double-stranded oligodeoxynucleotides: A study by ESI ion-trap mass spectrometry. Journal of the American Chemical Society, 122, 300-307.

Young, N. D., Hall, R. S., Jex, A. R., Cantacessi, C., \& Gasser, R. B. (2010). Elucidating the transcriptome of Fasciola hepatica -A key to fundamental and biotechnological discoveries for a neglected parasite. Biotechnology Advances, 28, $222-231$.

Cite this article: Gouveia MJ, Pakharukova MY, Rinaldi G, Mordvinov VA, Brindley PJ, Gärtner F, Vale N (2020). Helminth infection-induced carcinogenesis: spectrometric insights from the liver flukes, Opisthorchis and Fasciola Experimental Results, 1, e40, 1-14. https://doi.org/10.1017/exp.2020.38 


\section{Peer Reviews}

\section{Reviewing editor: Prof. Martin Michaelis}

University of Kent, School of Biosciences, Canterbury, United Kingdom of Great Britain and Northern Ireland, CT2 7NJ

This article has been accepted because it is deemed to be scientifically sound, has the correct controls, has appropriate methodology and is statistically valid, and met required revisions.

doi:10.1017/exp.2020.38.pr1

Review 1: Helminth infection-induced carcinogenesis: spectrometric insights from the liver flukes, Opisthorchis and Fasciola

Reviewer: Dr. Sirikachorn Tangkawatana (D)

Date of review: 30 June 2020

(C) The Author(s), 2020. Published by Cambridge University Press This is an Open Access article, distributed under the terms of the Creative Commons Attribution licence (http://creativecommons.org/licenses/by/4.0/), which permits unrestricted re-use, distribution, and reproduction in any medium, provided the original work is properly cited.

Conflict of interest statement. Reviewer declares none.

Comments to the Author: This works is designed to compare the spectrometric profiles of soluble extracts from F. hepatica, O. viverrini and O. felineus. The authors found 3 common compounds: oxysterol-like metabolites, bile acids and DNA-adducts.

They believe the first compound related to carcinogenesis. The findings are interesting and could explain the different/ common pathogenesis caused by these flukes. However some points should be revised or clarified.

- Fig. 2 : Because the carcinogenesis is multistage-multistep process, other important factors should be added in the diagram.

- Does the host spp. affect the results? F. hepatica adult worms are from natural host but O. viverrini and $\mathrm{O}$. felineus worms are from animal model.

\section{Score Card}

Presentation

Is the article written in clear and proper English? (30\%)

Is the data presented in the most useful manner? (40\%)

Does the paper cite relevant and related articles appropriately? (30\%)

Context

Does the title suitably represent the article? (25\%)

Does the abstract correctly embody the content of the article? (25\%)

Does the introduction give appropriate context? (25\%)

Is the objective of the experiment clearly defined? (25\%) 
Analysis

Is the conclusion consistent with the results and discussion? (40\%)

Are the limitations of the experiment as well as the contributions of the experiment clearly outlined? (20\%) 
Review 2: Helminth infection-induced carcinogenesis: spectrometric insights from the liver flukes, Opisthorchis and Fasciola

Reviewer: Dr. Lydia Leonardo (D)

Date of review: 17 July 2020

(C) The Author(s), 2020. Published by Cambridge University Press This is an Open Access article, distributed under the terms of the Creative Commons Attribution licence (http://creativecommons.org/licenses/by/4.0/), which permits unrestricted re-use, distribution, and reproduction in any medium, provided the original work is properly cited.

Conflict of interest statement. Reviewer declares none.

Comments to the Author: Please discuss the basis for suspected association between fascioliasis and carcinogenesis. Include the initial similarity in life processes and metabolism shown by Fasciola spp and Opisthorchis spp before they diverge with the Opisthorchis becoming carcinogenic and the Fasciola otherwise. Take note of the comments cited in the manuscript.

\section{Score Card}

Presentation

Is the article written in clear and proper English? (30\%)

Is the data presented in the most useful manner? (40\%)

Does the paper cite relevant and related articles appropriately? (30\%)

\section{Context}

Does the title suitably represent the article? (25\%)

Does the abstract correctly embody the content of the article? (25\%)

Does the introduction give appropriate context? (25\%)

Is the objective of the experiment clearly defined? (25\%)

Analysis

Is the conclusion consistent with the results and discussion? (40\%)

Are the limitations of the experiment as well as the contributions of the experiment clearly outlined? $(20 \%)$ 